\title{
The Investigation of Solutions to the Coupled Schrödinger-Boussinesq Equations
}

\author{
Xin Huang ${ }^{1,2}$ \\ ${ }^{1}$ College of Mathematics and Software Science, Sichuan Normal University, Chengdu 610066, China \\ ${ }^{2}$ Department of Basic Courses, Sichuan Finance and Economics Vocational College, Chengdu 610101, China
}

Correspondence should be addressed to Xin Huang; huangxinnv@163.com

Received 12 May 2013; Accepted 2 June 2013

Academic Editor: Shaoyong Lai

Copyright (c) 2013 Xin Huang. This is an open access article distributed under the Creative Commons Attribution License, which permits unrestricted use, distribution, and reproduction in any medium, provided the original work is properly cited.

The $\left(G^{\prime} / G\right)$-expansion method and the symbolic computation system Mathematica are employed to investigate the coupled Schrödinger-Boussinesq equations. The hyperbolic function solutions, trigonometric function solutions, and rational function solutions to the equations are obtained. The decaying properties of several solutions are analyzed.

\section{Introduction}

In laser and plasma physics, the important problems under interactions between a nonlinear complex Schrödinger field and a real Boussinesq field have been raised. In particular, the study of the coupled Schrödinger-Boussinesq equations has attracted much attention of mathematicians and physicists (see [1-3]). The existence of the global solution of the initialboundary problem for the equations was investigated in [1]. The existence of a periodic solution for the equations was considered in [2]. Kilicman and Abazari [3] used the $\left(G^{\prime} / G\right)$ expansion method to construct periodic and soliton solutions for the Schrödinger-Boussinesq equations $i u_{t}+u_{x x}-a u v=0$, $v_{t t}-v_{x x}+v_{x x x x}-b\left(|u|^{2}\right)_{x x}=0$, where $a$ and $b$ are real constants. The investigation of nonlinear partial differential equations plays an important role in the study of nonlinear physical phenomena (see [4-7]).

In this paper, we consider the following coupled Schrödinger-Boussinesq equations:

$$
\begin{gathered}
i E_{t}+E_{x x}+\beta_{1} E=N E \\
3 N_{t t}-N_{x x x x}+3\left(N^{2}\right)_{x x}+\beta_{2} N_{x x}=\left(|E|^{2}\right)_{x x},
\end{gathered}
$$

where $E(x, t)$ is a complex unknown function, $N(x, t)$ is a real unknown function, and $\beta_{1}$ and $\beta_{2}$ are real positive constants. System (1) is known to describe various physical processes in laser and plasma physics, such as formation, Langmuir field amplitude, intense electromagnetic waves, and modulational instabilities (see [8]). The approximate solutions and conservation law for the coupled system (1) have been studied in [9]. In [10], Chen and $\mathrm{Xu}$ used the $F$-expansion method to obtain a number of periodic wave solutions expressed by various Jacobi elliptic functions for (1). Cai et al. [11] studied same equations by the modified $F$ expansion method.

In the present paper, we use the $\left(G^{\prime} / G\right)$-expansion method and the symbolic computation system Mathematica to investigate the coupled Schrödinger-Boussinesq system (1). Here, we state that the previous works do not obtain the solutions presented in this paper.

The layout of this paper is as follows. In Section 2, we give the description of the generalized $\left(G^{\prime} / G\right)$-expansion method. In Section 3, we apply this method to solve (1). A conclusion will be obtained in Section 4 .

\section{Brief Description of the $\left(G^{\prime} / G\right)$-Expansion Method}

To make our presentation self-contained, we recall the $\left(G^{\prime} / G\right)$-expansion method. The details can be found in Wang et al's work [12]. 
Step 1. For a given PDE with two independent variables $t$ and $x$

$$
P\left(u, u_{t}, u_{x}, u_{t t}, u_{x t}, u_{x x}, \ldots\right)=0,
$$

we convert it into an ODE

$$
P\left(u, u^{\prime}, u^{\prime \prime}, u^{\prime \prime \prime}, \ldots\right)=0 .
$$

Using travelling transformation $u(x, t)=u(\xi), \xi=x-k t$. Equation (3) can be integrated as long as all terms contain derivatives where integration constants are considered to be zeros.

Step 2. Suppose that the solution of (3) can be expressed as a polynomial in $\left(G^{\prime} / G\right)$

$$
u(\xi)=\sum_{i=0}^{n} a_{i}\left(\frac{G^{\prime}}{G}\right)^{i},
$$

where $G=G(\xi)$ satisfies the second-order ODE with respect to $\xi$. Namely,

$$
G^{\prime \prime}+\lambda G^{\prime}+\mu G=0,
$$

where $a_{1}, \ldots, a_{n} \neq 0, \lambda$, and $\mu$ are constants to be determined later. The positive integer $n$ can be determined by balancing the highest-order derivatives with highest-order nonlinear terms appearing in (3). It is easy to check that (5) admits three types of solutions

$$
\frac{G^{\prime}}{G}=\left\{\begin{array}{rr}
\frac{\sqrt{\alpha}}{2}\left(\frac{c_{1} \sinh (1 / 2) \sqrt{\alpha} \xi+c_{2} \cosh (1 / 2) \sqrt{\alpha} \xi}{c_{1} \cosh (1 / 2) \sqrt{\alpha} \xi+c_{2} \sinh (1 / 2) \sqrt{\alpha} \xi}\right)-\frac{\lambda}{2}, \\
\alpha>0, \\
\frac{\sqrt{-\alpha}}{2}\left(\frac{-c_{1} \sin (1 / 2) \sqrt{-\alpha} \xi+c_{2} \cos (1 / 2) \sqrt{-\alpha} \xi}{c_{1} \cos (1 / 2) \sqrt{-\alpha} \xi+c_{2} \sin (1 / 2) \sqrt{-\alpha} \xi}\right)-\frac{\lambda}{2}, \\
\frac{c_{2}}{c_{1}+c_{2} \xi}-\frac{\lambda}{2}, & \alpha=0,
\end{array}\right.
$$

in which $\alpha=\lambda^{2}-4 \mu$.

Step 3. By substituting (4) into (3) and using (5), collecting all terms with the same order of $\left(G^{\prime} / G\right)$ together, the left-hand side of (3) can be written as a polynomial in $\left(G^{\prime} / G\right)$. Letting each coefficient of this polynomial be zero yields a system of algebraic equations for $a_{1}, \ldots, a_{n}, k, \lambda$, and $\mu$.

Step 4. Since the general solutions of (5) have been known, substituting $a_{i}, \ldots, a_{n}, k, \lambda$, and $\mu$ into (4), we can obtain travelling wave solutions of the nonlinear evolution equation (2).

\section{Solutions of the Coupled \\ Schrödinger-Boussinesq Equations}

Following the procedure described in Section 2, we adopt the ansatz solution of (1) in the form

$$
E(x, t)=u(x, t) e^{i\left(k x+l t+\xi_{0}\right)},
$$

where $u(x, t)$ is a real function, $k, l$ are constants to be determined, and $\xi_{0}$ is an arbitrary constant. Substituting (7) into (1) yields

$$
\begin{gathered}
u_{t}+2 k u_{x}=0, \\
u_{x x}-\left(l+k^{2}-\beta_{1}\right) u=N u, \\
3 N_{t t}-N_{x x x x}+3\left(N^{2}\right)_{x x}+\beta_{2} N_{x x}=\left(u^{2}\right)_{x x} .
\end{gathered}
$$

We take

$$
u(x, t)=u(\xi)=u\left(x-2 k t+\xi_{1}\right)
$$

where $\xi_{1}$ is an arbitrary constant. Substituting (11) into (9), one gets

$$
N(x, t)=\frac{u^{\prime \prime}(\xi)}{u(\xi)}-\left(l+k^{2}-\beta_{1}\right) .
$$

Suppose that

$$
N(x, t)=v(\xi)=v\left(x-2 k t+\xi_{1}\right) .
$$

It follows from (9), (10), (11), and (13) that

$$
\begin{gathered}
u^{\prime \prime}-\left(l+k^{2}-\beta_{1}\right) u-u v=0, \\
-v^{\prime \prime}+\left(12 k^{2}+\beta_{2}\right) v+3 v^{2}-u^{2}=0 .
\end{gathered}
$$

Balancing $u^{\prime \prime}$ with $u v$ in (14) and $v^{\prime \prime}$ with $u^{2}$ in (15) leads to $m=1, n=2$. Thus we can search for the solutions of (14) and (15) in the following forms:

$$
\begin{array}{ll}
u(\xi)=a_{0}+a_{1}\left(\frac{G^{\prime}}{G}\right)+a_{2}\left(\frac{G^{\prime}}{G}\right)^{2}, & a_{2} \neq 0, \\
v(\xi)=b_{0}+b_{1}\left(\frac{G^{\prime}}{G}\right)+b_{2}\left(\frac{G^{\prime}}{G}\right)^{2}, & b_{2} \neq 0 .
\end{array}
$$

Substituting (16) and (17) into (14) and (15), using (5), and setting the coefficients of $\left(G^{\prime} / G\right)^{i}(i=0, \ldots, 4)$ to be zero, 
we obtain the algebraic system

$$
\begin{gathered}
6 a_{2}-a_{2} b_{2}=0 \\
2 a_{1}+10 a_{2} \lambda-a_{2} b_{1}-a_{1} b_{2}=0 \\
8 a_{2} \mu+3 a_{1} \lambda+4 a_{2} \lambda^{2}-l b_{2}-k^{2} b_{2} \\
+\beta_{1} b_{2}-a_{2} b_{0}-a_{1} b_{1}-a_{0} b_{2}=0 \\
6 a_{2} \lambda \mu+2 a_{1} \mu+a_{1} \lambda^{2}-l b_{1}-k^{2} b_{1}+\beta_{1} b_{1}-a_{1} b_{0}-a_{0} b_{1}=0 \\
2 a_{2} \mu^{2}+a_{1} \lambda \mu-l b_{0}-k^{2} b_{0}+\beta_{1} b_{0}-a_{0} b_{0}=0 \\
6 b_{2}-3 b_{2}^{2}+a_{2}^{2}=0 \\
2 b_{1}+10 b_{2} \lambda-6 b_{1} b_{2}+2 a_{1} a_{2}=0 \\
8 b_{2} \mu+3 b_{1} \lambda+4 b_{2} \lambda^{2}-12 k^{2} b_{2}+\beta_{2} b_{2} \\
-3 b_{1}^{2}+6 b_{0} b_{2}+a_{1}^{2}+2 a_{0} a_{2}=0 \\
6 b_{2} \lambda \mu+2 b_{1} \mu+b_{1} \lambda^{2}-12 k^{2} b_{1}-\beta_{2} b_{1}-6 b_{0} b_{1}+2 a_{0} a_{1}=0 \\
2 b_{2} \mu^{2}+b_{1} \lambda \mu-12 k^{2} b_{0}-\beta_{2} b_{0}-3 b_{0}^{2}+a_{0}^{2}=0
\end{gathered}
$$

Solving this system with the Mathematica, we find

$$
\begin{gathered}
a_{0}= \pm\left(\sqrt{2} \lambda^{2}+2 \sqrt{2} \mu\right), \\
a_{1}= \pm 6 \sqrt{2} \lambda, \\
a_{2}= \pm 6 \sqrt{2}, \\
b_{0}=\lambda^{2}+2 \mu, \\
b_{1}=6 \lambda, \\
b_{2}=6, \\
k= \pm \frac{1}{6} \sqrt{3\left(\beta_{2}+\lambda^{2}-4 \mu\right),} \\
l=\frac{1}{12}\left(12 \beta_{1}+\beta_{2}+\lambda^{2} \mp 12 \sqrt{2} \lambda^{2}-4 \mu \pm 48 \sqrt{2} \mu\right),
\end{gathered}
$$

or

$$
\begin{gathered}
a_{0}= \pm 6 \sqrt{2} \mu, \\
a_{1}= \pm 6 \sqrt{2} \lambda, \\
a_{2}= \pm 6 \sqrt{2}, \quad b_{0}=6 \mu, \\
b_{1}=6 \lambda, \\
b_{2}=6, \\
k= \pm \frac{1}{6} \sqrt{3\left(\beta_{2}-\lambda^{2}+4 \mu\right)} \\
l=\frac{1}{12}\left(12 \beta_{1}+\beta_{2}-\lambda^{2} \pm 12 \sqrt{2} \lambda^{2}+4 \mu \mp 48 \sqrt{2} \mu\right),
\end{gathered}
$$

where $\lambda$ and $\mu$ are arbitrary constants.
By using (19) and (20), the solutions (16) and (17) are written as

$$
\begin{gathered}
u(\xi)= \pm\left(\sqrt{2} \lambda^{2}+2 \sqrt{2} \mu\right) \pm 6 \sqrt{2} \lambda\left(\frac{G^{\prime}}{G}\right) \pm 6 \sqrt{2}\left(\frac{G^{\prime}}{G}\right)^{2} \\
v(\xi)=\lambda^{2}+2 \mu+6 \lambda\left(\frac{G^{\prime}}{G}\right)+6\left(\frac{G^{\prime}}{G}\right)^{2},
\end{gathered}
$$

where $\xi=x \pm(1 / 3) \sqrt{3\left(\beta_{2}+\lambda^{2}-4 \mu\right)} t+\xi_{1}$, or

$$
\begin{gathered}
u(\xi)= \pm 6 \sqrt{2} \mu \pm 6 \sqrt{2} \lambda\left(\frac{G^{\prime}}{G}\right) \pm 6 \sqrt{2}\left(\frac{G^{\prime}}{G}\right)^{2}, \\
v(\xi)=6 \mu+6 \lambda\left(\frac{G^{\prime}}{G}\right)+6\left(\frac{G^{\prime}}{G}\right)^{2},
\end{gathered}
$$

where $\xi=x \pm(1 / 3) \sqrt{3\left(\beta_{2}-\lambda^{2}+4 \mu\right)} t+\xi_{1}$.

Substituting general solutions of (5) into (21) and (22), we obtain three types of travelling wave solutions of the coupled Schrödinger-Boussinesq equations as follows.

$$
\begin{aligned}
& \text { 3.1. The Hyperbolic Function Solutions to (1) If } \alpha=\lambda^{2}-4 \mu>0 . \\
& \text { Consider } \\
& \begin{aligned}
E_{1}(x, t) \\
= \pm \\
\quad \times \frac{\sqrt{2} \alpha}{2} \\
\quad \times\left[3\left(\frac{c_{1} \sinh (1 / 2) \sqrt{\alpha} \xi+c_{2} \cosh (1 / 2) \sqrt{\alpha} \xi}{c_{1} \cosh (1 / 2) \sqrt{\alpha} \xi+c_{2} \sinh (1 / 2) \sqrt{\alpha} \xi}\right)^{2}-1\right] \\
\quad \times e^{i\left(k x+l t+\xi_{0}\right)}, \\
N_{1}(x, t) \\
=\frac{\alpha}{2}\left[3\left(\frac{c_{1} \sinh (1 / 2) \sqrt{\alpha} \xi+c_{2} \cosh (1 / 2) \sqrt{\alpha} \xi}{c_{1} \cosh (1 / 2) \sqrt{\alpha} \xi e g+c_{2} \sinh (1 / 2) \sqrt{\alpha} \xi}\right)^{2}-1\right],
\end{aligned}
\end{aligned}
$$

where $k= \pm(1 / 6) \sqrt{3\left(\beta_{2}+\alpha\right)}, \quad l=(1 / 12)\left(12 \beta_{1}+\beta_{2}+\lambda^{2} \mp\right.$ $12 \sqrt{2} \lambda^{2}-4 \mu \pm 48 \sqrt{2} \mu, c_{1}, c_{2}$ are arbitrary constants, and

$E_{2}(x, t)$

$$
\begin{aligned}
= & \pm \frac{3 \sqrt{2} \alpha}{2} \\
& \times\left[\left(\frac{c_{1} \sinh (1 / 2) \sqrt{\alpha} \xi+c_{2} \cosh (1 / 2) \sqrt{\alpha} \xi}{c_{1} \cosh (1 / 2) \sqrt{\alpha} \xi+c_{2} \sinh (1 / 2) \sqrt{\alpha} \xi}-1\right]\right. \\
& \times e^{i\left(k x+l t+\xi_{0}\right)} \\
N_{2}(x, t) & \\
= & \frac{3 \alpha}{2}\left[\left(\frac{c_{1} \sinh (1 / 2) \sqrt{\alpha} \xi+c_{2} \cosh (1 / 2) \sqrt{\alpha} \xi}{c_{1} \cosh (1 / 2) \sqrt{\alpha} \xi+c_{2} \sinh (1 / 2) \sqrt{\alpha} \xi}\right)^{2}-1\right],
\end{aligned}
$$


where $k= \pm(1 / 6) \sqrt{3\left(\beta_{2}-\alpha\right)}, l=(1 / 12)\left(12 \beta_{1}+\beta_{2}-\lambda^{2} \pm\right.$ $\left.12 \sqrt{2} \lambda^{2}+4 \mu \mp 48 \sqrt{2} \mu\right)$.

Remark 1. When $\xi \rightarrow \infty$, we find $\left|E_{1}(x, t)\right| \rightarrow \pm \sqrt{2} \alpha$, $N_{1}(x, t) \rightarrow \alpha,\left|E_{2}(x, t)\right| \rightarrow 0, N_{2}(x, t) \rightarrow 0$.

Remark 2. If $c_{1} \neq 0, c_{2}=0, \lambda>0, \mu=0$, we find envelope solitary wave solutions for (1). Namely, $E_{1}(x, t)$ and $N_{1}(x, t)$ become

$$
\begin{gathered}
E_{1}(x, t)= \pm \frac{\sqrt{2}}{2} \lambda^{2}\left(3 \tanh ^{2} \frac{\lambda}{2} \xi-1\right) e^{i\left(k x+l t+\xi_{0}\right)}, \\
N_{1}(x, t)=\frac{1}{2} \lambda^{2}\left(3 \tanh ^{2} \frac{\lambda}{2} \xi-1\right) .
\end{gathered}
$$

$E_{2}(x, t)$ and $N_{2}(x, t)$ are turned into

$$
\begin{gathered}
E_{2}(x, t)= \pm \frac{3 \sqrt{2}}{2} \lambda^{2}\left(\tanh ^{2} \frac{\lambda}{2} \xi-1\right) e^{i\left(k x+l t+\xi_{0}\right)} \\
N_{2}(x, t)=\frac{3}{2} \lambda^{2}\left(\tanh ^{2} \frac{\lambda}{2} \xi-1\right) .
\end{gathered}
$$

3.2. The Trigonometric Function Solutions to (1) If $\alpha<0$. Consider

$$
\begin{aligned}
& E_{3}(x, t) \\
& = \pm \frac{\sqrt{2} \alpha}{2} \\
& \quad \times\left[3\left(\frac{-c_{1} \sin (1 / 2) \sqrt{-\alpha} \xi+c_{2} \cos (1 / 2) \sqrt{-\alpha} \xi}{c_{1} \cos (1 / 2) \sqrt{-\alpha} \xi+c_{2} \sin (1 / 2) \sqrt{-\alpha} \xi}\right)^{2}+1\right] \\
& \quad \times e^{i\left(k x+l t+\xi_{0}\right)}, \\
& N_{3}(x, t) \\
& =-\frac{\alpha}{2}\left[3\left(\frac{-c_{1} \sin (1 / 2) \sqrt{-\alpha} \xi+c_{2} \cos (1 / 2) \sqrt{-\alpha} \xi}{c_{1} \cos (1 / 2) \sqrt{-\alpha} \xi+c_{2} \sin (1 / 2) \sqrt{-\alpha} \xi}\right)^{2}+1\right],
\end{aligned}
$$

where $k= \pm(1 / 6) \sqrt{3\left(\beta_{2}+\alpha\right)}, l=(1 / 12)\left(12 \beta_{1}+\beta_{2}+\lambda^{2} \mp\right.$ $\left.12 \sqrt{2} \lambda^{2}-4 \mu \pm 48 \sqrt{2} \mu\right)$, and

$$
\begin{aligned}
& E_{4}(x, t) \\
& = \pm \frac{3 \alpha}{2} \\
& \times \quad\left[\left(\frac{-c_{1} \sin (1 / 2) \sqrt{-\alpha} \xi+c_{2} \cos (1 / 2) \sqrt{-\alpha} \xi}{c_{1} \cos (1 / 2) \sqrt{-\alpha} \xi+c_{2} \sin (1 / 2) \sqrt{-\alpha} \xi}\right)^{2}+1\right] \\
& \quad \times e^{i\left(k x+l t+\xi_{0}\right)}, \\
& N_{4}(x, t) \\
& =-\frac{3 \alpha}{2}\left[\left(\frac{-c_{1} \sin (1 / 2) \sqrt{-\alpha} \xi+c_{2} \cos (1 / 2) \sqrt{-\alpha} \xi}{c_{1} \cos (1 / 2) \sqrt{-\alpha} \xi+c_{2} \sin (1 / 2) \sqrt{-\alpha} \xi}\right)^{2}+1\right],
\end{aligned}
$$

where $k= \pm(1 / 6) \sqrt{3\left(\beta_{2}-\alpha\right)}, l=(1 / 12)\left(12 \beta_{1}+\beta_{2}-\lambda^{2} \pm\right.$ $\left.12 \sqrt{2} \lambda^{2}+4 \mu \mp 48 \sqrt{2} \mu\right)$.

3.3. The Rational Function Solutions to (1) If $\alpha=0$. We obtain

$$
\begin{gathered}
E_{5}(x, t)= \pm \frac{6 \sqrt{2} c_{2}^{2}}{\left(c_{1}+c_{2} \xi\right)^{2}} e^{i\left(k x+l t+\xi_{0}\right)}, \\
N_{5}(x, t)=\frac{6 c_{2}^{2}}{\left(c_{1}+c_{2} \xi\right)^{2}},
\end{gathered}
$$

where $k= \pm(1 / 6) \sqrt{3\left(\beta_{2}+\alpha\right)}, l=(1 / 12)\left(12 \beta_{1}+\beta_{2}+\lambda^{2} \mp\right.$ $\left.12 \sqrt{2} \lambda^{2}-4 \mu \pm 48 \sqrt{2} \mu\right)$, and

$$
\begin{gathered}
E_{6}(x, t)=E_{9,10}(x, t)= \pm \frac{6 \sqrt{2} c_{2}^{2}}{\left(c_{1}+c_{2} \xi\right)^{2}} e^{i\left(k x+l t+\xi_{0}\right)}, \\
N_{6}(x, t)=N_{9,10}(x, t)=\frac{6 c_{2}^{2}}{\left(c_{1}+c_{2} \xi\right)^{2}},
\end{gathered}
$$

where

$$
\begin{gathered}
k= \pm \frac{1}{6} \sqrt{3\left(\beta_{2}-\alpha\right)}, \\
l=\frac{1}{12}\left(12 \beta_{1}+\beta_{2}+\lambda^{2} \pm 12 \sqrt{2} \lambda^{2}+4 \mu \mp 48 \sqrt{2} \mu\right) .
\end{gathered}
$$

\section{Conclusion}

The $\left(G^{\prime} / G\right)$-expansion method is effectively employed to deal with the coupled Schrödinger-Boussinesq equations. The hyperbolic function solutions, the trigonometric function solutions, and the rational function solutions to the equations in the case of $\alpha>0, \alpha<0$, and $\alpha=0$ are obtained. In particular, the well-known soliton solutions are only the special case of the hyperbolic-type solutions. We find several properties of solutions when $\xi \rightarrow \infty$.

\section{Acknowledgments}

This work is supported by the National Natural Science Foundation of China (11171241, 11071177, 11226162), the Key Project of Chinese Ministry of Education (Grant no. 211162), and the Sichuan Province Science Foundation for Youths (no. 2012JQ0011).

\section{References}

[1] B. L. Guo and L. J. Shen, "The global solution of initial value problem for nonlinear Schrödinger-Boussinesq equation in 3dimensions," Acta Mathematicae Applicatae Sinica, vol. 6, no. 1, pp. 11-21, 1990.

[2] B. Guo and X. Du, "Existence of the periodic solution for the weakly damped Schrödinger-Boussinesq equation," Journal of Mathematical Analysis and Applications, vol. 262, no. 2, pp. 453472, 2001.

[3] A. Kilıcman and R. Abazari, "Travelling wave solutions of the Schrödinger-Boussinesq system," Abstract and Applied Analysis, vol. 2012, Article ID 198398, 11 pages, 2012. 
[4] S. Y. Lai and B. Wiwatanapataphe, "The asymptotics of global solutions for semilinear wave equations in two space dimensions," Dynamics of Continuous, Discrete \& Impulsive Systems. Series B, vol. 18, no. 5, pp. 647-657, 2011.

[5] S. Lai, Y. H. Wu, and B. Wiwatanapataphee, "On exact travelling wave solutions for two types of nonlinear $K(n, n)$ equations and a generalized KP equation," Journal of Computational and Applied Mathematics, vol. 212, no. 2, pp. 291-299, 2008.

[6] S. Lai and Y. Wu, "The local well-posedness and existence of weak solutions for a generalized Camassa-Holm equation," Journal of Differential Equations, vol. 248, no. 8, pp. 2038-2063, 2010.

[7] S. Lai and A. Wang, "The well-posedness of solutions for a generalized shallow water wave equation," Abstract and Applied Analysis, vol. 2012, Article ID 872187, 15 pages, 2012.

[8] H. Schamel and K. Elsässer, "The application of the spectral method to nonlinear wave propagation," Journal of Computational Physics, vol. 22, no. 4, pp. 501-516, 1976.

[9] V. G. Makhankov, “On stationary solutions of Schrödinger equation with a self-consistent potential satisfying Boussinesq's equations," Physics Letters A, vol. 50, no. 1, pp. 42-44, 1974.

[10] H. L. Chen and Z. H. Xu, "Periodic wave solutions for the coupled Schrödinger-Boussinesq equations," Acta Mathematicae Applicatae Sinica, vol. 29, no. 5, pp. 955-960, 2006.

[11] G. L. Cai, F. Y. Zhang, and L. Ren, "More exact solutions for coupling Schrödinger-Boussinesq equations by a modified $F$ expansion method," Mathematica Applicata, vol. 21, no. 1, pp. 90-97, 2008.

[12] M. Wang, X. Li, and J. Zhang, “The $\left(G^{\prime} / G\right)$-expansion method and travelling wave solutions of nonlinear evolution equations in mathematical physics," Physics Letters A, vol. 372, no. 4, pp. 417-423, 2008. 


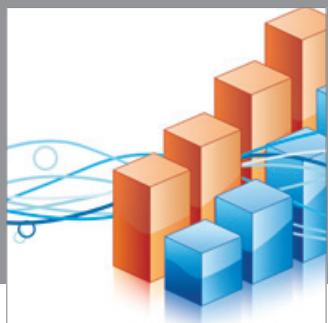

Advances in

Operations Research

mansans

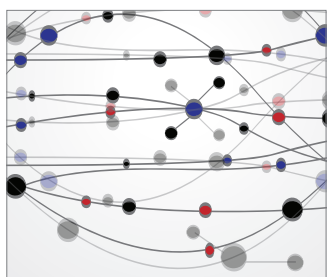

The Scientific World Journal
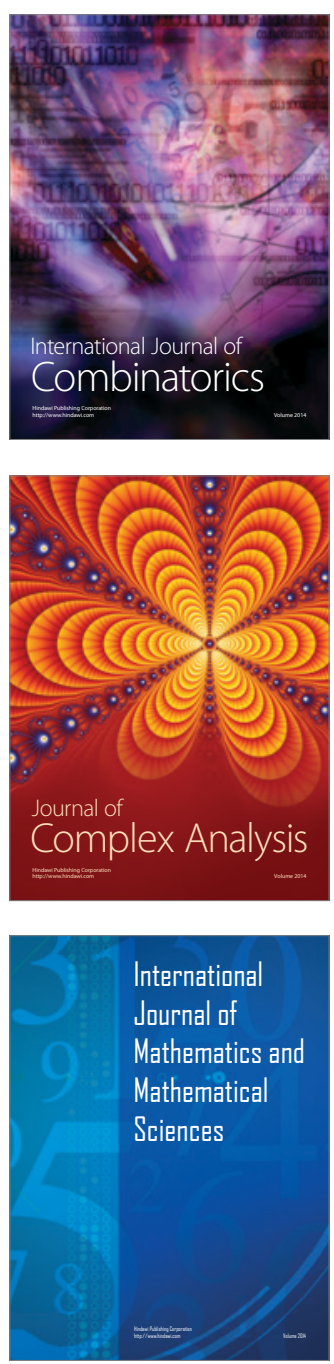
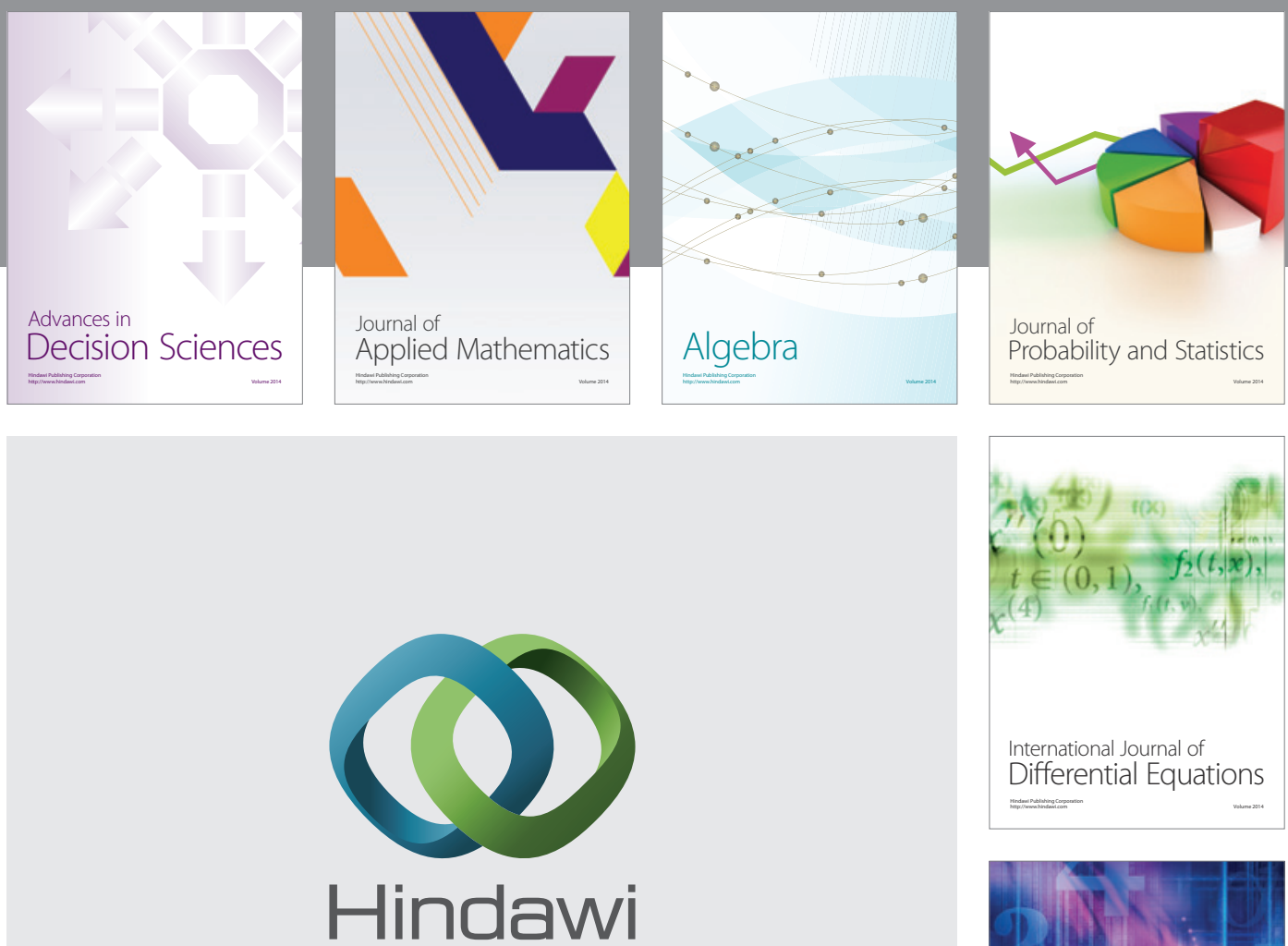

Submit your manuscripts at http://www.hindawi.com
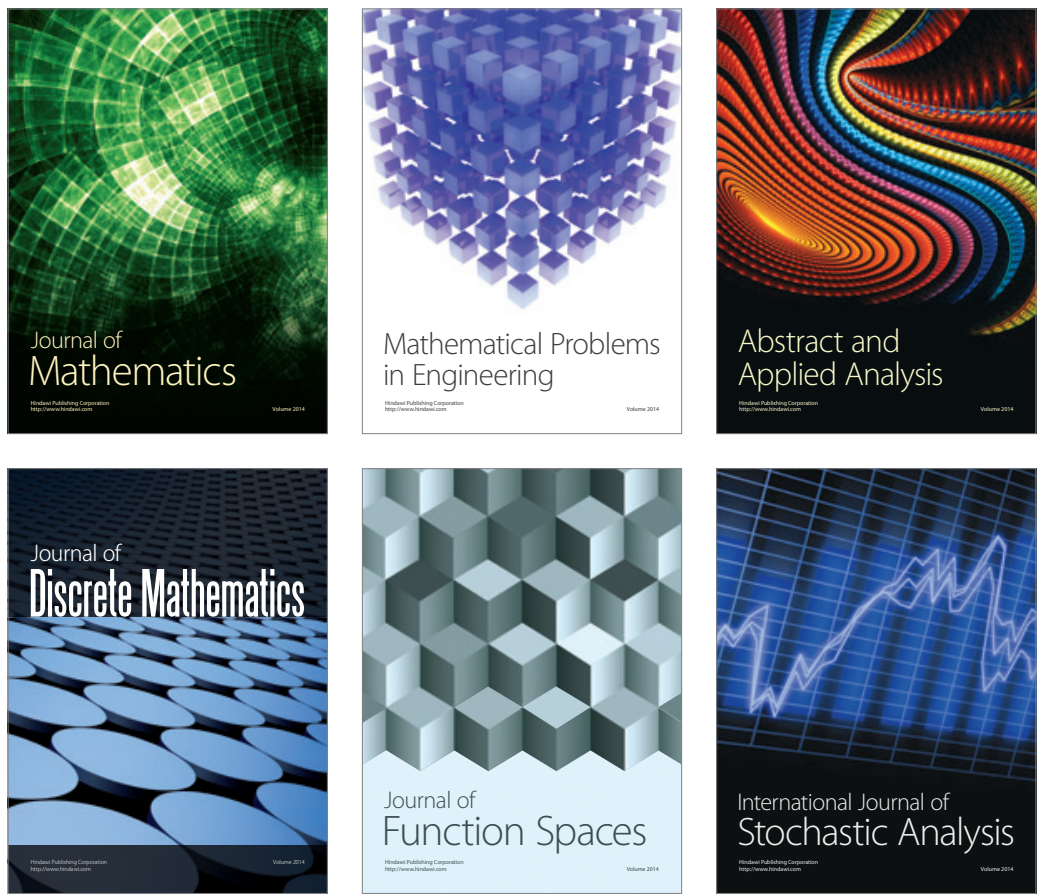

Journal of

Function Spaces

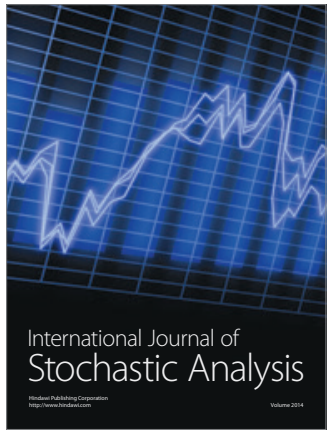

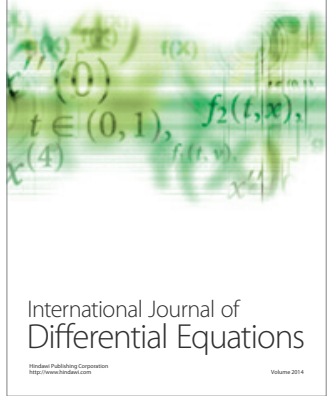
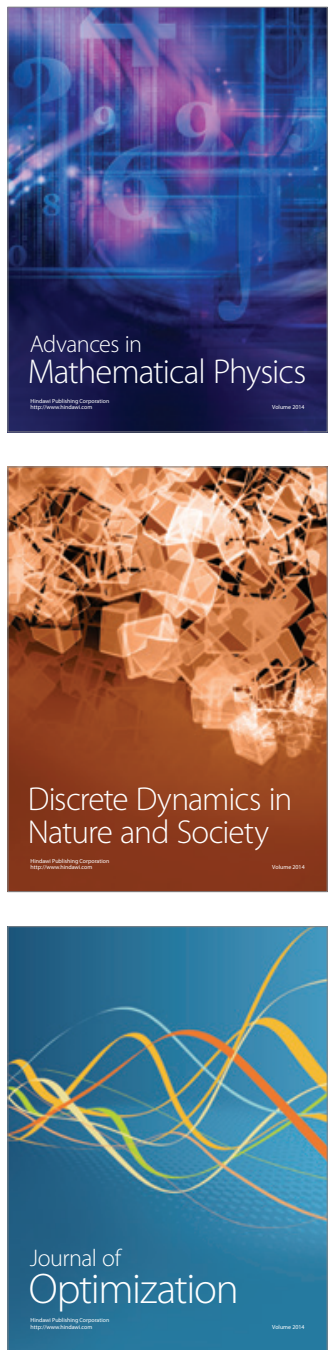